\title{
On type IIB vacua with varying coupling constant
}

\author{
P. Berglund ${ }^{a}$, A. Klemm ${ }^{\text {a,b }}$, P. Mayr ${ }^{a, c}$, S. Theisen ${ }^{a, d}$ \\ a Institute for Theoretical Physics, University of California, Santa Barbara, CA 93106, USA \\ ${ }^{\mathrm{b}}$ Enrico Fermi Institute, University of Chicago, Chicago, IL 60637, USA \\ ' Theory Division, CERN, 1211 Geneva 23, Switzerland \\ d Sektion Physik, Universität München, D-80333 Munich, Germany
}

Received 30 June 1998; revised 2 July 1999; accepted 15 July 1999

\begin{abstract}
We describe type IIB compactifications with varying coupling constant in $d=6,7,8,9$ dimensions, where part of the ten-dimensional $S L(2, \mathbb{Z})$ symmetry is broken by a background with $\Gamma_{1}(n)$ or $\Gamma(n)$ monodromy for $n=2,3,4$. This extends the known class of F-theory vacua to theories which are dual to heterotic compactifications with reduced rank. On compactifying on a further torus, we obtain a description of the heterotic moduli space of $G$ bundles over elliptically fibered manifolds without vector structure in terms of complex geometries. (C) 1999 Published by Elsevier Science B.V. All rights reserved.
\end{abstract}

PACS: 11.25.-w

Keywords: F-theory; CHL strings

\section{Introduction}

F-theory [1] provides an interesting dual description of heterotic strings in various dimensions. Amongst the most promising aspects is the fact that some non-geometric data of the heterotic string are included in the geometric description of the F-theory compactification. A large class of F-theory/heterotic duals has been described in [26]. ${ }^{1}$ The purpose of this note is to extend this dictionary to a large class of heterotic theories with extra $U(1)$ backgrounds. This includes CHL type of vacua [8] in various dimensions as well as $U(1)$ instanton backgrounds.

Let us recall the crucial ingredients of the construction in [1]. Type IIB theory in ten dimensions has a conjectured strong/weak coupling symmetry $\Gamma_{0}=S L(2, \mathbb{Z})$. $\bar{\Gamma}_{0}=\operatorname{PSL}(2, \mathbb{Z})$ acts on the complex dilaton field $\tau=\phi+i \exp (\tilde{\phi})$ [9] by fractional

\footnotetext{
${ }^{1}$ For a detailed one-loop comparison in $8 \mathrm{~d}$, see Ref. [7]. 
linear transformations. $\Gamma_{0}$ acts also non-trivially on the two-form potentials $B^{N S}$ and $B^{R R}$ of the theory while leaving the other bosonic fields invariant. The idea of [1] is to interpret the complex parameter $\tau$ as the modulus of a complex torus $E$ and consider type IIB compactifications on a base manifold $B$ with varying $\tau$. Since the monodromy group of the moduli space of the torus $E$ is generically $\Gamma_{0}, \tau$ will jump by an $\bar{\Gamma}_{0}$ transformation at those points $b \subset B$ on the base, where the torus $E$ degenerates. Due to the $\Gamma_{0}$ symmetry of type IIB this is nevertheless a valid vacuum. In physical terms, the points where $\tau \rightarrow i \infty$ are to be identified as D7-branes of the type IIB theory. The type IIB equations of motion require the total manifold $X_{n}$ of the fiber $E$ together with the $n-1$ dimensional base $B_{n-1}$ to be a Calabi-Yau manifold. By construction, it has an elliptic fibration $\pi: X_{n} \rightarrow B_{n-1}$.

Note that generically, the ten-dimensional $\Gamma_{0}$ symmetry is completely broken by the non-trivial vacuum due to the fact that the monodromy of $E$ generates all of $\Gamma_{0}$. In particular, the BPS states, in a given $\Gamma_{0}$ orbit of BPS states, of the ten-dimensional type IIB theory are transformed into each other by the action of the $\Gamma_{0}$ monodromy.

It is natural to extend this construction by compactifications which do not generate all of the $\bar{\Gamma}_{0}$ transformations acting on $\tau$. This does not necessarily mean that there is a left-over symmetry group of the lower-dimensional theory corresponding to a non-trivial commutant $H$ of a subgroup $\Gamma \subseteq \Gamma_{0}$. Instead, we consider the case where $\Gamma$ is a finite index subgroup of $\Gamma_{0}$ with trivial commutant $H$. Upon compactification, each $\Gamma_{0}$ orbit of BPS states of the ten-dimensional type IIB theory splits into several orbits of the coset $\Gamma_{0} / \Gamma$ leading to a very different spectrum in the lower-dimensional theory. This is comparable to the situation in $N=4$ and $N=2$ Super-Yang-Mills theories in four dimensions: in the $N=4$ theory with a $\Gamma_{0}$ strong-weak coupling duality, magnetic and electric degrees of freedom can be equivalent due to the fact that they are living in a single $\Gamma_{0}$ multiplet. In the $N=2$ theory with a $\Gamma \subset \Gamma_{0}$ symmetry, there are two very different kinds of short multiplets corresponding to the vector multiplets and the monopole hypermultiplets. The charges of the two types of multiplets are related by the "missing" transformations ${ }^{2}$ in $\Gamma_{0} / \Gamma$. Note that the distinct BPS spectra of the theories with different symmetry groups rule out a continuous interpolation from one theory to the other at finite distance in moduli space.

What we find is that the type IIB compactifications with restricted monodromy on $\tau$ lead to moduli spaces describing Calabi-Yau manifolds with frozen moduli. These restricted geometric moduli spaces, which have a natural interpretation in terms of toric geometry as we will see, correspond to heterotic compactifications with frozen moduli via F-theory/heterotic duality. In the heterotic theory the freezing can be either due to a CHL type modding or due to $U(1)$ instanton backgrounds. A type IIB interpretation for the freezing in the case of monodromy group $\Gamma_{0}(2)$ has been described in six-and eight-dimensional orientifold constructions: it corresponds to the choice of a non-trivial $\mathbb{Z}_{2}$ background for the Neveu-Schwarz two-form $B_{\mu \nu}$. In particular, non-triviality of the $B_{\mu \nu}$ background is related to the absence of vector structure in the type I SO(32)

\footnotetext{
${ }^{2}$ For example, for pure $N=2 S U(2)$ theory the subgroup is $I_{0}(2)$.
} 
string [10] which in turn corresponds to a reduced rank compactification described by a CHL vacuum [11]. The choice of a $\mathbb{Z}_{2}$ valued $B$ field is compatible with the restricted monodromy group $\Gamma_{0}(2)$. There appears to be one additional non-trivial choice apart from a $\mathbb{Z}_{2}$ valued $B$ field in eight dimensions, namely the case $\mathbb{Z}_{4}$. The other case that we discuss, $\Gamma_{1}(3)$, cannot be pushed to a higher than seven-dimensional vacuum.

The paper is organized as follows. In Section 2 we study F-theory compactifications with the elliptic fiber of a less generic type, i.e. with $\Gamma \subset \Gamma_{0}$ monodromy. We then turn to the gauge enhancements and the relevant dual CHL heterotic theories in Section 3, and in particular when there exists an F-theory limit to eight dimensions. The heterotic interpretation naturally gives the moduli space of $G$ bundles without vector structure on elliptic curves, as discussed in Section 4.

While this work was being finished for publication we received a preprint by Bershadsky, Pantev and Sadov [12], which, to a certain extent, overlaps with our results.

\section{Type IIB geometries with $\Gamma \subset \Gamma_{0}=S L(2, \mathbb{Z})$ monodromy}

To define a type IIB compactification with varying coupling constant along the lines of Ref. [1], we give an elliptically fibered Calabi-Yau manifold $X_{n}$ of complex dimension $n$ together with a section $\sigma$, on which the type IIB string is compactified. ${ }^{3}$ Let $E$ denote the elliptic fiber and $B_{n-1}$ the base of the fibration $X \rightarrow B$. A model for $E$ and $X_{n}$ can be given in terms of (an intersection of) hypersurfaces in a projective space (or generalizations thereof). As we will explain in a moment, the model for $E$ will determine the monodromy group $\bar{\Gamma} \subset \bar{\Gamma}_{0}$ acting on the complex structure $\tau$ of $E$ in the fibration. In particular, $\Gamma$ being contained in $\Gamma_{1}(n)$ is related to the presence of shift symmetries of finite order of the elliptic fiber $E$. Specifically, $\Gamma_{1}(n)(\Gamma(n))$ preserves $n\left(n^{2}\right)$ points of order $n$ on $E$ pointwise.

\subsection{Fiber geometry and fibrations over $\mathbf{P}^{1}$}

To be concrete, we will consider models of elliptic curves $E$ given as the zero of one or two polynomials of appropriate degree in weighted projective space and elliptic K3 surfaces with $E$ as the generic fiber. The K3 case contains all the essential features also for the case of higher-dimensional Calabi-Yau manifolds with restricted monodromies, which can be treated very similarly.

\subsubsection{Generic case: $\Gamma_{0}$ monodromy}

Let us consider a model of a generic elliptic curve $E$ given as the zero of a polynomial of degree six in weighted projective space $\mathbf{P}_{1,2,3}^{2}$,

$$
E^{(1)}: \quad y^{2}+x^{3}+z^{6}+\mu x z^{4}=0, \quad(z, x, y) \in \mathbf{P}_{1,2,3}^{2} .
$$

\footnotetext{
${ }^{3}$ The situation of F-theory on manifolds without section is unclear. This interesting issue is beyond the scope of this paper.
} 
To get an elliptically fibered $\mathrm{K} 3$ with $E^{(1)}$ as the generic fiber, we fiber $E^{(1)}$ over a base $\mathbf{P}^{1}$ by making the coefficients of the monomials in $E^{(1)}$ functions ${ }^{4}$ of the base coordinates $(s, t)$ of $\mathbf{P}^{1}$ in a way which is compatible with the Calabi-Yau condition. In this way we get a K3 surface

$$
X_{2}^{(1)}: \quad y^{2}+x^{3}+x z^{4} f_{8}+z^{6} f_{12}=0
$$

where $f_{l}$ are homogeneous polynomials of degree $l$ in the base variables $(s, t)$. To be more precise the coordinates $(z, x, y, s, t)$ (with $s=t=0$ and $z=x=y=0$ excluded) are identified by the $\left(\mathbb{C}^{*}\right)^{2}$-actions $(z, x, y, s, t) \sim\left(\lambda \rho^{-2} z, \lambda^{2} x, \lambda^{3} y, \rho s, \rho t\right)$ with $\lambda, \rho \in \mathbb{C}^{*}$. At $z=0$, the elliptic fibrations has one section, because $y^{2}+x^{3}=0$ has, in view of the coordinate indentifications, one solution.

The elliptic fiber of $X_{2}^{(1)}$ has the generic $\Gamma_{0}$ monodromy, and F-theory compactified on $X_{2}^{(1)}$ is dual to the standard heterotic compactification on $T^{2}$ in eight dimensions with gauge group $G$ of rank 18 [1]. The zeros of the discriminant $\delta=4 f_{8}^{3}+27 f_{12}^{2}=0$ are identified with the locations of $\mathbf{D} 7$-branes on the $\mathbf{P}^{\mathbf{l}}$ base. For generic $f_{8}$ and $f_{12}$ there are 24 simple zeros corresponding to singular elliptic fibers. The total manifold is nevertheless smooth since a singularity in the K3 would correspond to a zero of order $\geqslant 2$ in $\delta$. The independent number of D7-brane positions determining the rank of $G$ in the F-theory compactification is given by the $13+9-4=18$ independent parameters in (2.2), where we had to subtract 3 parameters for the reparametrizations of the base coordinates and one overall scaling.

\subsubsection{Type IIB backgrounds with $\Gamma_{1}(2)$ and $\Gamma(2)$ monodromy}

Instead of the model (2.1) for $E$, we consider a degree four polynomial in $\mathbf{P}_{1,1,2}^{2}$. The generic polynomial has 9 terms, but the 8 parameters in the redefinitions of $(x, y, z)$ compatible with the weights allow us to choose a simple form for the defining equation

$$
E^{(2)}: \quad y^{2}+x^{4}+x^{2} z^{2} \mu+z^{4}=0, \quad(x, z, y) \in \mathbf{P}_{1,1,2}^{2} .
$$

In order to remove the dependence on the choice of representative, the monodromy subgroup is usually obtained by considering

$$
\hat{\jmath}(\mu)=\frac{\left(12+\mu^{2}\right)^{3}}{108\left(\mu^{2}-4\right)^{2}}=j(\tau),
$$

where $\hat{j}(\mu)$ is invariant under changes on the coordinates $(x, y, z)$ and $j(\tau)$ is the $\bar{\Gamma}_{0}$ invariant function. Eq. (2.4) does not quite imply that $\mu(\tau)$ is $\bar{\Gamma}_{0}$ invariant. The involutions $m: \mu \mapsto-\mu$ and $n: \mu \mapsto \frac{2 \mu+12}{\mu-2}$ leave $\hat{\jmath}(\mu)$ invariant. They satisfy $(m n)^{3}=1$ and thus generate $S_{3}$, which can be identified with the group permuting the roots of (2.4), read as a sixth order polynomial in $\mu$. The monodromy group is then $\Gamma(2)$ with $\vec{\Gamma}_{0} / \bar{\Gamma}(2) \simeq S_{3}$.

However, given the preferred representation (2.3) there is an easier way to see the monodromy group by noting that $E^{(2)}$ enjoys two $\mathbb{Z}_{2}$ symmetries generated by

\footnotetext{
${ }^{4}$ More precisely sections of $\mathcal{O}_{\mathbf{p}^{\prime}}(l)$.
} 


$$
\mathcal{S}_{1}:(y, x, z) \rightarrow(-y, z, x), \quad \mathcal{S}_{2}:(y, x, z) \rightarrow(-y,-x, z) .
$$

It can be verified that the symmetries $\mathcal{S}_{i}$ act without fixed points and leave the holomorphic one-form invariant. A symmetry of the elliptic curve translates to a symmetry of the lattice $\Lambda$ spanned by the periods, where $E$ is defined as $E=C / \Lambda$. In particular, fixed-point free $\mathbb{Z}_{n}$ symmetries must be order $n$ shift symmetries on $E$. The presence of an order $n$ shift implies that there are $n$ points in a finer lattice, so-called points of order $n$, which are equivalent to the origin up to a $\mathbb{Z}_{n}$ shift. The monodromy does not change the symmetries and preserves the order $n$ points and is therefore a subgroup of $\Gamma_{0}(n)$. If it fixes the order $n$-points pointwise it is actually $\Gamma_{1}(n)$, cf. Appendix A. Similarly, two independent $\mathbb{Z}_{n}$ shifts, corresponding to a finer lattice in the two directions of $A$, imply the existence of $n^{2}$ such points and the monodromy group $\Gamma(n)$. Below we will fiber tori with known modular groups and by fibering we can only lower the monodromy to a subgroup of the above.

To fibre the elliptic curve (2.3) over $\mathbf{P}^{1}$ we consider an $\mathbf{P}^{2}(1,1,2)$ bundle over $\mathbf{P}^{1}$, defined by the $\mathbb{C}^{*}$-actions $(z, x, y, s, t) \sim\left(\lambda \rho^{-2} z, \lambda x, \lambda^{2} y, \rho s, \rho t\right)$. The generic elliptically fibred $K 3$ hypersurface is then given by $y^{2}+x^{4}+x^{2} z^{2} f_{4}+x z^{3} f_{6}+z^{4} f_{8}=0$ and one sees at $z=0$ "two" sections, because $y^{2}+x^{4}=0$ has two inequivalent solutions. ${ }^{6}$ This $K_{3}$ has 17 deformations and Picard number 3. To get fibrations with restricted monodromy we specialize to the shift invariant form $E^{(2)}$ by setting $f_{6}=0$ :

$$
X_{2}^{(2)}: \quad y^{2}+x^{4}+x^{2} z^{2} f_{4}+z^{4} f_{8}=0 .
$$

As now one $\mathbb{Z}_{2}$ shift is realized, the monodromy preserves the order 2 points and must therefore a subgroup of $\Gamma_{0}(2)$. The Weierstrass form $\left(y^{2}=4 x^{3}-g_{2} x-g_{3}\right)$ of the generic hypersurface factorizes precisely if $f_{6}=0$

$$
y^{2}=\left(4 x+\frac{4 f_{4}}{\sqrt{3}}\right)\left(x^{2}-\frac{f_{4}}{\sqrt{3}} x-3 f_{8}+\frac{f_{4}^{2}}{12}\right) \text {. }
$$

This shows that the second section is now at an order 2 point at $(x, y)=\left(-\frac{f_{4}}{\sqrt{3}}, 0\right)$, i.e. at one of the half-periods (cf. Appendix A). This point is hence fixed, which is consistent with the $\Gamma_{0}(2)$ monodromy. Note that $\Gamma_{1}(2) \sim \Gamma_{0}(2)$. The singularities of the restricted form, which generate this monodromy are listed in Appendix B.

As before, we can determine the rank of the gauge group $G$ by counting the number of independent parameters in the polynomial; this time we get $5+9-4=10$. Thus, compactification of F-theory on $X_{2}^{(2)}$ is dual to heterotic compactification on $T^{2}$ with a rank reduction of -8 as compared to the standard heterotic compactification. This is characteristic of the original CHL compactification, which can be understood as a compactification on $T^{2}$ which combines a half-shift on a circle of $T^{2}$ with an exchange

\footnotetext{
${ }^{5}$ One section can be taken to at zero with respect to the group law on $E$. The other is then at an undistinguished position with respect to the group law and generates infinitely many sections, however only two section are inequivalent in homology.

${ }^{6}$ The phenomenon that an ambient space divisor meets the surface $n$-times manifests itself in the toric polyhedra diagrams via the occurrence of points of multiplicity $n$; cf. Section 3.4 .
} 
of the two $E_{8}$ factors in the $E_{8} \times E_{8}$ heterotic string or the exchange of two $S O$ (16) factors in the $S O(16) \times S O(16) \subset S O(32)$ of the $S O(32)$ heterotic string [13]. ${ }^{7}$ As for the D7-brane locations, the discriminant of the elliptic fibration $X_{2}^{(2)}$ is given by $\delta=f_{8}\left(4 f_{8}-f_{4}^{2}\right)^{2}$. Thus, we have eight simple zeros which correspond to eight dynamical D7-branes as well as eight double zeros corresponding to $A_{1}$ singularities of the manifold $X_{2}^{(2)}$.

The $j$-function of the elliptic fibration $X_{2}^{(2)}$ is

$$
j=\frac{1}{108} \frac{\left(f_{4}^{2}+12 f_{8}\right)^{3}}{f_{8}\left(f_{4}^{2}-4 f_{8}\right)^{2}} .
$$

We will justify the identification of F-theory on $X_{2}^{(2)}$ and the existence of a eightdimensional limit in the next section, where we explore the geometrical properties of $X_{2}^{(2)}$.

We can restrict the monodromy further to $\Gamma(2) \subset \Gamma_{1}(2)$ by choosing $f_{8}=\hat{f}_{4}^{2}$. We then obtain a rank $10-4=6$ theory which should correspond to a heterotic theory with rank reduced by -12 . The corresponding heterotic CHL vacua in six dimensions have also been constructed in [15]. The singularity structure is $\left(A_{1}\right)^{12}$. Moreover, the Weierstrass form (2.7) factorizes further corresponding to four global sections of the elliptic fibration.

\subsubsection{Type IIB backgrounds with $\Gamma_{1}(3)$ and $\Gamma$ (3) monodromy}

In order to get a monodromy restricted to $\Gamma_{1}(3)$, we consider an elliptic curve $E^{(3)}$ given as a degree three polynomial in $\mathbf{P}_{1,1,1}^{2}$ :

$$
E^{(3)}: \quad y^{3}+x^{3}+z^{3}+\mu y x z=0, \quad(y, x, z) \in \mathbf{P}_{1,1,1}^{2} .
$$

This torus enjoys two $\mathbb{Z}_{3}$ shift symmetries generated by

$$
\begin{aligned}
& \mathcal{S}_{1}:(y, x, z) \rightarrow(x, z, y), \\
& \mathcal{S}_{2}:(y, x, z) \rightarrow\left(y, \lambda x, \lambda^{2} z\right), \quad \lambda=e^{2 \pi i / 3} .
\end{aligned}
$$

The monodromy group in this case is $\Gamma(3)$ with $\bar{\Gamma}_{0} / \bar{\Gamma}(3) \simeq A_{4}$.

In the $\mathbf{P}^{2}$ fibration over $\mathbf{P}^{1}$ defined by the $\mathbb{C}^{*}$-actions

$$
(x, y, z, s, t) \sim\left(\lambda \rho^{-2} z, \lambda x, \lambda y, \rho s, \rho t\right)
$$

the general elliptically fibred $K 3$ is given by $y^{3}+y^{3}+f_{2} x y z+f_{4} y z^{2}+f_{4}^{\prime} x z^{2}+f_{6} x^{3}=0$ and has at $z=0$ "three" sections. Similarly as before, we specialize to K3 with $E^{(3)}$ as elliptic fiber:

$$
X_{2}^{(3)}: \quad y^{3}+x^{3}-y x z f_{2}+z^{3} f_{6}=0 .
$$

Now the presence of the $Z_{3}$-symmetry implies that for the special from, two sections lie on the $\frac{1}{3}$ - and $\frac{2}{3}$-period points, which are fixed under the monodromy. This can

\footnotetext{
${ }^{7}$ A more detailed analysis of this case has appeared in $[14]$.
} 
also been seen by relating (2.11) to the $Z_{3}$ torsion normal form (cf. Appendix B) and implies that the monodromy is restricted to $\Gamma_{1}(3)$. The monodromy generated by the singularities of the specialized form confirms that.

Counting the number of independent parameters we get $3+7-4=6$ for the rank of the gauge group. We will identify this theory in the next section with a CHL type construction, where we first compactify the heterotic $S O(32)$ string on $T^{2}$ to get an $S O(36)$ gauge group, and then combine a $\mathbb{Z}_{3}$ shift on a further $T^{2}$ with a permutation of the three $S O(12)$ factors in $S O(12)^{3} \subset S O(36)$ to get a six-dimensional vacuum with reduction of rank -12 as compared to the standard compactification [15]. The discriminant has the form $\delta=f_{6}\left(f_{2}^{3}-27 f_{6}\right)^{3}$. Thus, in this case we have six simple zeros corresponding to six dynamical D7-branes as well as six triple zeros corresponding to $A_{2}$ singularities of $X_{2}^{(3)}$.

If we further restrict $f_{6}$ to be the cube of a degree two polynomial $\hat{f}_{2}$, we obtain an elliptic fibration with $\Gamma(3)$ monodromy. The singularity structure is $\left(A_{2}\right)^{8}$ and the number of remaining parameters is $3+3-4=2$, corresponding to a rank -16 reduction on the heterotic side. A CHL vacuum with this properties has been given in [16]

We can also look for points in the moduli space where the type IIB coupling $\tau$ becomes constant as in Ref. [17]. In particular such a configuration would be a possible candidate for an orientifold vacuum of type IIB. The $j$-function of the elliptic fibration of $X_{2}^{(3)}$ reads

$$
j=\frac{1}{1728} \frac{f_{2}^{3}\left(216 f_{6}+f_{2}^{3}\right)^{3}}{f_{6}\left(f_{2}^{3}-27 f_{6}\right)^{3}} .
$$

A constant coupling limit which intersects weak type IIB coupling requires $f_{6} \sim f_{2}^{3}$. However, for this choice $X_{2}^{(3)}$ acquires a $\hat{E}_{8} \times \hat{E}_{8}$ singularity which has no valid interpretation as a weakly coupled orientifold.

\subsubsection{Type IIB backgrounds with $\Gamma_{1}(4)$ and $\Gamma(4)$ monodromy}

Restricted $\Gamma_{1}(4)$ monodromy can be obtained by choosing a complete intersection in $\mathbf{P}^{3}$ as a model for the elliptic fiber $E$ :

$$
E^{(4)}: \quad u^{2}+v^{2}+\mu x z=0, \quad x^{2}+z^{2}+\mu u v=0, \quad(u, v, x, z) \in \mathbf{P}^{3} .
$$

This torus has two $\mathbb{Z}_{4}$ shift symmetries generated by

$$
\begin{aligned}
& \mathcal{S}_{1}:(u, v, x, z) \rightarrow(x, z, v, u), \\
& \mathcal{S}_{2}:(u, v, x, z) \rightarrow\left(\lambda u, \lambda^{-1} v, \lambda^{2} x, z\right), \quad \lambda=e^{2 \pi i / 4} .
\end{aligned}
$$

The monodromy group is $\Gamma(4)$ with $\bar{\Gamma}_{0} / \bar{\Gamma}(4) \simeq S_{4}$. As before, we embed the complete intersection in an $\mathbf{P}^{3}$ bundle over $\mathbf{P}^{1}$ with $\mathbb{C}^{*}$-actions

$$
(u, v, x, z, s, t) \sim\left(\lambda u, \lambda v, \lambda x, \lambda \rho^{-2} z, \rho s, \rho t\right)
$$


and excluded loci $s=t=0$ and $u=v=x=z=0$. At $z=0$ it has 4 "four" sections and hence Picard group 5 and 15 deformations. The $\mathrm{K} 3$ with $E^{(4)}$ as the generic elliptic fiber:

$$
X_{2}^{(4)}: \quad u^{2}+v^{2}+x z f_{2}=0, \quad x^{2}+z^{2} f_{4}+u v=0
$$

has the $Z_{4}$ shift symmetry and hence by similar arguments as before $\Gamma_{1}(4)$ monodromy. Counting the number of independent parameters we get $3+5-4=4$ for the rank of the gauge group. We suggest to identify this theory with a CHL type construction in eight dimensions, where the four factors $S O(8)^{4} \subset S O(32)$ are permuted together with a $\mathbb{Z}_{4}$ shift on $T^{2}$ [15]. The discriminant of the elliptic fibration $X_{2}^{(4)}$ is $\delta=f_{2}^{2} f_{4}\left(f_{4}-\frac{1}{16} f_{2}^{2}\right)^{4}$. Thus, in this case we have 4 simple zeros corresponding to four dynamical D7-branes as well as 2 double zeros and 4 zeroes of order 4 corresponding to $A_{1}^{2} \times A_{3}^{4}$ singularities of $X_{2}^{(4)}$.

The $j$-function for the elliptic fibration $X_{2}^{(4)}$ is

$$
j=\frac{1}{1728} \frac{\left(f_{2}^{4}+224 f_{4} f_{2}^{2}+256 f_{4}^{2}\right)^{3}}{f_{2}^{2} f_{4}\left(f_{2}^{2}-16 f_{4}\right)^{4}} .
$$

It can be verified that the Weierstrass form factorizes indicating a second section as expected.

Restricting $f_{4}=\hat{f}_{2}^{2}$ we get an elliptic fibration with $\left(A_{1}\right)^{4} \times\left(A_{3}\right)^{4}$ singularity and $6-4=2$ parameters. Moreover, for $\hat{f}_{2} \sim f_{2}$ we get a configuration with constant coupling. As in the case of $\Gamma(3)$ however, the singularity structure in this limit is $\hat{E}_{8} \times \hat{E}_{8}$ which cannot be interpreted as an orientifold. $\Gamma(4)$ can only be recovered if $f_{4}=f_{1}^{4}$ and $f_{2}=\tilde{f}_{1}^{2}$. In this case we have $2+2-4=0$, i.e. no moduli left.

The cases with special monodromies have also additional sections. For an eightdimensional F-theory interpretation we must be able to declare a $\mathbf{P}^{\mathbf{l}}$ section of the elliptic fibration as the space the theory actually lives on, this will be discussed in the next section.

\subsection{Rank reduction in F-theory}

In the F-theory picture the rank of the gauge group in eight dimensions is usually 18. This is because one needs 24 D7-branes to make the transverse space compact, however only $18 \mathrm{D} 7$-branes can be chosen to have the same $(p, q)$ charge under the $\Gamma_{0}$ of type IIB and are therefore independent [1]. The D7-branes are located at points on the section of the elliptic fibration which is isomorphic to the $\mathbf{P}^{\mathbf{l}}$ base of the elliptic K3 surface. This is the space the type IIB string is compactified on. To reduce the rank, we somehow need to get rid of some of the D7-branes. It turns out that the F-theory geometries with restricted monodromy find a clever way to achieve this: we will find that there are two sections, where on the first one we have the usual fiber singularities corresponding to a reduced rank gauge group of the type IIB theory in eight dimensions, whereas on the other section we have singularities which account for the missing rank 
in the eight-dimensional theory. The first section is the one on which type IIB theory is compactified to eight dimensions while we have to contract the second section. So the rank reduction is due to the fact that the type IIB theory sees only the D7-branes on the section on which it is compactified; these generate the reduced rank gauge group present in eight dimensions.

To demonstrate this mechanism we can follow a deformation of the generic K3 surface to the one relevant for the $\Gamma_{1}(2)$ theory dual to the heterotic CHL vacuum. We start with the generic non-singular K3 surface $\mathbf{P}_{1,1,4,6}^{3}[12]$ with generic elliptic fiber $\mathbf{P}_{1,2,3}^{2}[6]$ :

$$
p_{A}=y^{2}+x^{3}+x z^{4} f_{8}+z^{6} g_{12},
$$

where $f_{8}$ and $g_{12}$ are polynomials of degree 8 and 12 in the base variables $s, t$, respectively. We have 18 independent complex deformations in $p_{A}$ and two scaling actions $(y, x, z, s, t) \rightarrow\left(\lambda^{3} y, \lambda^{2} x, \lambda \mu^{-2} z, \mu s, \mu t\right)$ representing the two Kähler classes generated by the fiber and a section.

In fact, (2.17) is a special choice of representative for the generic polynomial, due to the freedom to redefine variables compatible with the weights. A different representative is

$$
y^{2}+x^{3}+x z^{4} f_{8}+y z^{3} g_{6}+x^{2} z^{2} f_{4}
$$

By changing variables, $(2.18)$ can be put into the form (2.17) up to the fact that we get only 12 parameters for the polynomial $g_{12}$, that is $(2.18)$ describes a special codimension one subspace of the deformation (2.17). In addition to the canonical section $\sigma: z=0$ we have two more sections, namely $\tilde{\sigma}: x=y=0$ and $x=0, y=-g_{6}$. However, the new Kähler class associated to the volume of $\tilde{\sigma}$ can not be represented in the algebraic model $\mathbf{P}_{1,1,4,6}^{3}[12]$. This is because the toric ambient space $\mathbf{P}_{1,1,4,6}^{3}$ itself has only two Kähler classes which descend to the class of the elliptic fiber and the original section on the hypersurface. We can find a different model by introducing new variables via

$$
x \rightarrow x u, \quad y \rightarrow y u,
$$

and we get

$$
p_{B}=u y^{2}+u^{2} x^{3}+x z^{4} f_{8}+y z^{3} g_{6}+u x^{2} z^{2} f_{4} \text {. }
$$

This equation describes a different algebraic model in a toric ambient space with three Kähler classes. The new algebraic model $X_{B}$ describes now correctly the $\mathrm{K} 3$ surface with Picard lattice of rank 3 . There is also a new holomorphic elliptic class of type $\mathbf{P}_{1,1,2}^{2}$ [4] with variables $(y, z, u) \sim\left(\lambda y, \lambda z, \lambda^{2} u\right)$ with a volume that depends on the new Kähler class. Note that the two elliptic fibrations share the section $z=0$.

Up to now, all we have done is to restrict to a certain subspace in complex structure moduli space of the elliptic fibration. F-theory on $X_{B}$ does not give rise to a new eightdimensional theory since in the limit of small fiber the moduli spaces of $X_{A}$ and $X_{B}$ 
coincide. ${ }^{8}$ Therefore the deformation from $X_{A}$ to $X_{B}$ is a new branch only in the sevendimensional $\mathrm{M}$-theory compactification. This is related to the fact that the two elliptic fibrations in $X_{B}$ do not yet have restricted monodromy. In particular, the presence of the term $y z^{3} g_{6}$ term is not compatible with the shift symmetries of (2.3). Note that the original elliptic fibration with fiber coordinates $(y, x, z)$ has now two sections, $\sigma$ and $\tilde{\sigma}$. Moreover, $\left.X_{B}\right|_{g_{6}=0}$ has an $\left(A_{1}\right)^{8}$ singularity on the section $\tilde{\sigma}$, namely $y=x=f_{8}=0$.

To provide a single section on which type IIB theory is compactified, we contract $\tilde{\sigma}$. First we blow up the locus $y=x=0$ by introducing the coordinate $\hat{x}$ via

$$
y \rightarrow y \hat{x}, x \rightarrow x \hat{x}^{2}
$$

After this blow-up, the divisor $x=0$ no longer intersects the hypersurface $p_{C}=0$ which corresponds to a blow down of $\tilde{\sigma}$. Therefore we can set $x=1$ to get a new algebraic K3 model

$$
p_{C}=u y^{2}+u^{2} \hat{x}^{4}+y z u \hat{x} s t+u \hat{x}^{2} z^{2} f_{4}+z^{4} f_{8} .
$$

This algebraic K3 describes two elliptic fibrations with the quartic $E^{(2)}$ in $\mathbf{P}_{1,1,2}^{2}$ as a fiber model; they have coordinates $(\hat{x}, z, y)$ and $(y, z, u)$, respectively. The first fibration has the restricted $\Gamma_{1}(2)$ monodromy and describes the F-theory dual to the CHL vacuum in eight dimensions. The second fibration is not of the $\Gamma_{1}(2)$ type. ${ }^{9}$ This fibration corresponds to a CHL vacuum in six dimensions with $F_{4} \times F_{4}$ gauge symmetry as we will explain below.

It is easy to keep track of the blow-ups in a toric representation of the geometry. To this end, use the $\mathbb{C}^{*}$ actions to go to inhomogeneous coordinates (e.g. set $z=t=1$ ) and read the shifted exponents $\nu^{\text {mon }}=\left(n_{x}, n_{y}, n_{s}\right)$ of every monomial $y^{n_{y}+1} x^{n_{x}+1} s^{n_{s}+}$ in (2.17) as a vector in the lattice $A^{*}=\mathbb{Z}^{3}$ embedded in $N^{*}=\mathbb{R}^{3}$. The CalabiYau condition is equivalent to the requirement that the convex hull of all $\nu^{\text {mon }}$ is a reflexive polyhedron $\Delta^{*}=\operatorname{Conv}\left\{\nu^{\text {mon }}\right\}$. This means that the dual polyhedron $\Delta=\{x \in$ $\left.N \mid\langle x, y\rangle \geqslant-1, \forall y \in \Delta^{*}\right\}$ has only lattice points in $\Lambda$ as vertices. The polyhedron $\Delta=\operatorname{Conv}\left\{\nu^{\text {coord }}\right\}$ defines the ambient space, in our case $\mathbf{P}_{1,1,4,6}^{3}$, and the points $\nu^{\text {coord }}$ provide the coordinates such that the defining equation, in our case $(2.17)$, reads $[18,19]$

$$
p=\sum_{i} a_{i} \prod_{j} x_{j}^{\left\langle\nu_{i}^{\text {mon }}, \nu_{j}^{\text {courd }}\right\rangle+1}=0 .
$$

Here the sum runs over points $\nu_{i}^{\text {mon }}$ of $\Delta^{*}$ and the product over points $\nu_{j}^{\text {coord }}$ of $\Delta$. The fact that for the reflexive pair $\left(\Delta, \Delta^{*}\right)$ the rôle of $\Delta^{*}$ and $\Delta$ can be exchanged in (2.23) is the manifestation of mirror symmetry in Batyrev's construction. Generally, every reflexive pair of $d+1$-dimensional polyhedra $\left(\Delta, \Delta^{*}\right)$ defines in this way

\footnotetext{
${ }^{8}$ In particular, blowing down the original elliptic class $E^{(1)}$ in $X_{B}$ always blows down also the second class $E^{(2)}$. Therefore we simply end up at a special point in the complex structure moduli space of $X_{A}$ in the zero size limit.

${ }^{9}$ Because of the term $u y^{2}$.
} 


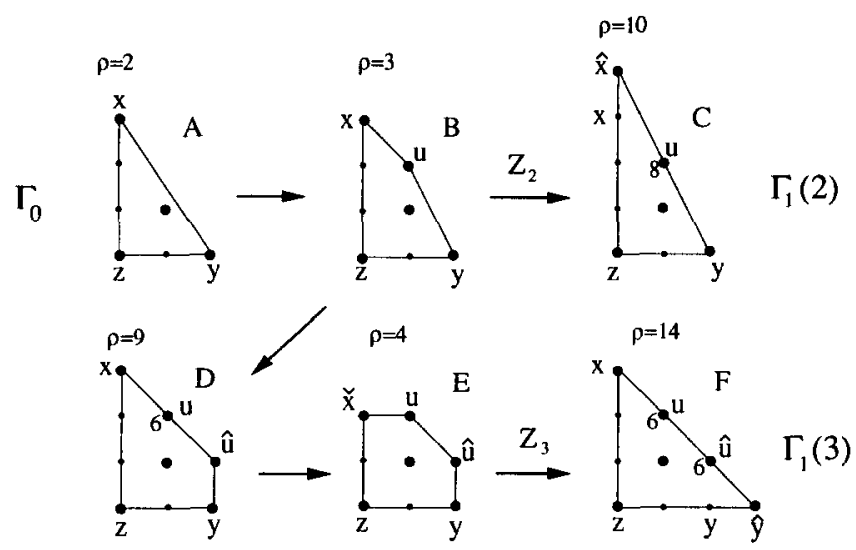

Fig. 1. The chains of blow-ups, which lead from a K3 with $\Gamma_{0}$ monodromy to such with $\Gamma_{1}(2)$ and $\Gamma_{1}(3)$ monodromy, $\rho=\operatorname{rank}(\operatorname{Pic}(X))$ and the origin is the interior point.

via (2.23) a $d$-dimensional Calabi-Yau manifold $X(d=2$ for $K 3)$ and its mirror ${ }^{10}$ $X^{*}$.

To be concrete, note that for (2.17) $\Delta=\operatorname{Conv}\left\{\nu^{\text {coord }}\right\}$ with

$$
\nu^{\text {coord }}=\{(-1,2,0),(1,-1,0),(-1,-1,0),(-1,-1,1),(-1,-1,-1)\}
$$

providing the coordinates $(x, y, z, s, t)$, while the allowed monomials in (2.17) correspond to the points in

$$
\Delta^{*}=\operatorname{Conv}\{(1,0,0),(0,1,0),(-2,-3,6),(-2,-3,-6)\} .
$$

Fibration structures can be detected from the toric diagrams by the fact that the reflexive polyhedron of the fiber is embedded in the polyhedron of the total space $[21,22]$. The elliptic fiber is represented by the two-dimensional reflexive sub-polyhedra $\Delta_{E}$ and $\Delta_{E}^{*}$ in the $(x, y, 0)$-planes of $\Delta$ and $\Delta^{*}$, respectively. $\Delta_{E}$ is shown in Fig. 1a and all blow-ups, some of them were discussed above, can be described by adding points in the $\Delta$-plane. The $K 3$ polyhedron $\Delta$ is completed by adding $\nu_{s}=(-1,-1,1)$ and $\nu_{t}=(-1,-1,-1)$ to $\Delta_{E}$.

For example, the blow-up (2.19) corresponds to adding the point $u$ with $\nu_{u}=\nu_{x}+\nu_{y}$ in 1.B, etc. $p_{B}, p_{C}$ are (2.23) written in the indicated coordinates. Spelling out $p_{D}, p_{E}, p_{F}$ can be done analogously. The lattice points indicated by small dots are irrelevant as they correspond to divisors of the ambient space which do not intersect the hypersurface. ${ }^{11}$ Therefore $x=0$ does not intersect $p_{C}=0$ and $y=0$ does not intersect $p_{F}=0$. The numbers labeling the points indicate how often $x_{i}=0$ intersects $p$ (if different from one). For example, $u=0$ intersects $p_{C}=0$ in the eight zeros of $f_{8}$ along a $\mathbf{P}^{1}$ and

\footnotetext{
${ }^{10}$ For $\mathrm{K} 3$ rank(Pic) of $X$ is exchanged with the dimension of the deformation space of the mirror [20] only if there is no correction term in Eq. (3.1) below.

${ }^{11}$ Because of $\nu_{s}, \nu_{t}$ they lie on a codimension one face and in the toric construction those divisors do not intersect $X$.
} 

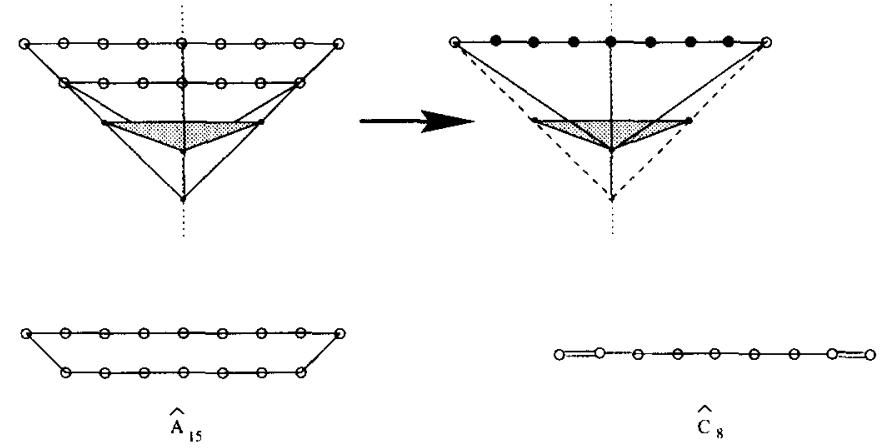

$\hat{C}_{8}$
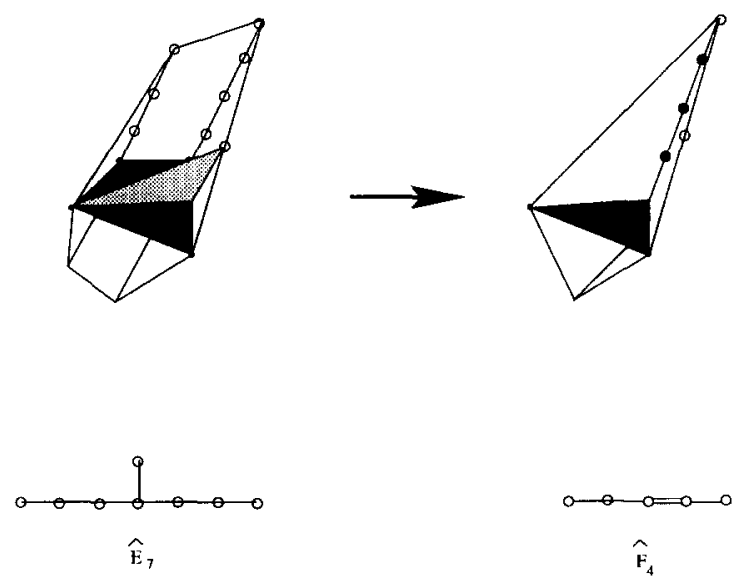

Fig. 2. Folding of K3 polyhedra for F-theory compactification.

represent the $A_{1}^{8}$ read from the discriminant in the previous section. Similarly, the sixfold divisors $\{u=0\} \cap\left\{p_{F}=0\right\}$ and $\{\hat{u}=0\} \cap\left\{p_{F}=0\right\}$ account for the $\left(A_{2}\right)^{6}$. The arrows with $\mathbb{Z}_{2}$ and $\mathbb{Z}_{3}$ correspond the orbifoldisation, whose action on the homology of $X^{*}$ is described in Figs. 2 and 3. When the divisors in the elliptic fiber $\Delta_{E}^{*}$ of $X^{*}$ are contracted, the IIA theory exhibits the following gauge groups: $\left(E_{8}\right)^{2},\left(E_{7}\right)^{2},\left(F_{4}\right)^{2}$, $\left(F_{4}\right)^{2},\left(E_{6}\right)^{2}$ and $\left(G_{2}\right)^{2}$. By blowing up $\hat{u}=y=0$ it is possible to pass from $D$ to $F$. However, in the $E$ model the $C H L \mathbb{Z}_{3}$ is realised as an outer automorphism on the cohomology lattice forming $\left(\hat{E}_{6}\right)^{2}$, see Fig. 3 .

\section{Gauge groups and heterotic duals}

\subsection{A type IIA perspective}

One way to learn about the possible enhanced non-abelian gauge symmetries of the above type IIB compactifications is to analyze the possible fiber degenerations of 


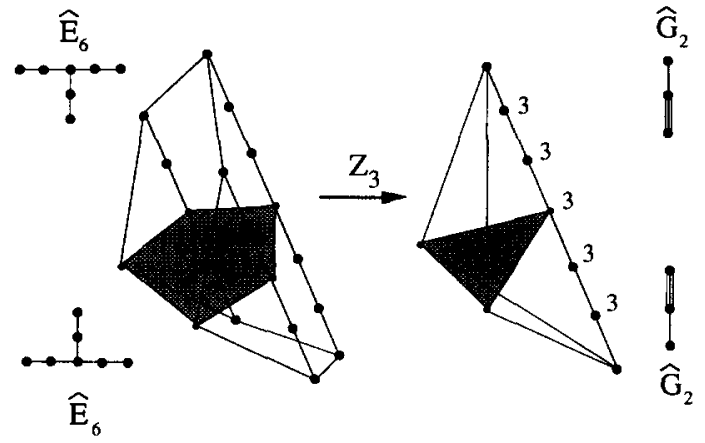

Fig. 3. $\mathbb{Z}_{3}$ folding of the $\hat{E}_{6} \times \hat{E}_{6} \mathrm{~K} 3$ to an elliptic fibration with $\Gamma_{1}(3)$ monodromy.

the manifolds described there, corresponding to collisions of D7-branes as in $[2,3]$. Alternatively, we can consider compactifying on a further $T^{2}$ to six dimensions which is dual to type IIA compactification on the same manifold, which is in turn dual to the heterotic string on $T^{4}$. The higher-dimensional case can be recovered by considering a limit of the K3 compactification where the elliptic fiber shrinks to zero size. In the type IIA compactification the gauge symmetry is largely determined by the homology lattice of 2-cycles. In particular, D2-brane states wrapped on the 2-cycles give rise to vector multiplets and the intersection lattice of small 2-cycles coincides generically with the Dynkin diagram of the enhanced non-abelian gauge symmetry [4,23]. However, the actual gauge symmetry can be smaller than what would be expected from the lattice of homology cycles, corresponding to the presence of certain $\mathrm{RR} U(1)$ backgrounds on top of the K3 geometry [24]. More precisely, the claim of [24] is that if an automorphism of the homology lattice of $\mathrm{K} 3$ is combined with a shift in a $U(1)$ factor from the $\mathrm{RR}$ sector, the actual gauge symmetry is given by the Dynkin diagram of the invariant homology lattice, corresponding to a folding of the original Dynkin diagram spanned by the full homology lattice. We will give further evidence for this proposal by realizing the appropriate restricted moduli spaces explicitly in terms of frozen parameters in toric geometries.

To see the connection with the higher-dimensional type IIB backgrounds above, recall that our fibers $E^{(i)}$ support the $n$th order shift symmetries $\mathcal{S}_{i}$. These shift symmetries will identify homology elements of the $\mathrm{K} 3$ manifold; thus the K3's we obtain from fibering symmetric tori with $\mathbb{Z}_{n}$ shift symmetries will have non-trivial $\mathbb{Z}_{n}$ automorphisms acting on the homology lattice. Finite abelian automorphisms of K3 have been classified in [25] and used in [15] to determine type IIA duals of CHL heterotic strings in six dimensions.

In order to be able to push these theories up to higher dimensions, the automorphism has to leave invariant a sub-lattice $\Gamma_{k, k}$ which is to be identified with $k$ circles of the heterotic $T^{4}$ that we decompactify. Thus, our type IIB theory with reduced monodromy group $\Gamma \subset \Gamma_{0}$ should be in one-to-one correspondence with finite automorphism groups of K3 lattices preserving a non-trivial $\Gamma_{k, k}$ lattice. In fact, it is not difficult to see, using the results in [25], that there are three automorphism of this kind which correspond 
to the three symmetric tori $E^{(i)}$ given above. In the following we will justify this identification by a careful study of the singularities of the geometries $X_{2}^{(i)}$.

A geometrical realization of the $\mathbb{Z}_{2}$, which gives rise to the $9 \mathrm{~d}$ CHL compactification, has been given in [26] and used to study $N=16 \mathrm{~d}$ and $N=24 \mathrm{~d}$ compactifications of the CHL string. These theories can be decompactified to $9 \mathrm{~d}$.

\subsection{Folding of toric polyhedra}

The appearance of non-simply laced gauge groups in the type IIB compactification with restricted monodromy group has a very clear interpretation when we represent the Calabi-Yau manifold $X_{2}$ in terms of toric polyhedra.

As we have seen at the end of Section 2.2, a K3 manifold $X_{2}$ and its mirror $X_{2}^{*}$ are described by a three-dimensional polyhedron $\Delta$ and a dual polyhedron $\Delta^{\star}$. The vertices $\nu_{i}$ of $\Delta$ correspond to divisors and are (taking into account the multiplicity) in one to one correspondence with the holomorphic curves in the Picard lattice of $X_{2}$.

Using F-theory/heterotic duality, it was observed in [4,5] and further explained in [5] that the points above and below the hyperplane $\Delta_{E}$ form the affine Dynkin diagram of the possible non-abelian gauge symmetry enhancements from collisions of fiber singularities. A microscopic explanation can be given in terms of D2-brane geometries in type IIA theory [23]: since points in $\Delta$ correspond to holomorphic curves, they give rise to states from D2-brane wrappings. D2-brane states on different 2-cycles $C_{1}, C_{2}$ will interact if they have non-vanishing intersection $C_{1} \cap C_{2} \neq \emptyset$. In the $\mathrm{K} 3$, mutual intersections of these 2-cycles correspond, roughly speaking, to the links (edges) in the toric polyhedron. Thus, a configuration of points in $\Delta$ forming the Dynkin diagram of a gauge group $G$ gives rise to a collection of intersecting D2-brane wrappings. These generate a nonabelian gauge symmetry $G$ in the space-time theory in the limit where the volume of these 2-cycles goes to zero.

\subsection{1. $\Gamma_{1}(2)$ monodromy}

Let us consider in detail the case with $\Gamma_{1}(2)$ monodromy corresponding to the eightdimensional $\mathrm{CHL}$ vacuum with rank reduction -8 . We start with the mirror $X_{B}^{*}$ of the $\mathrm{K} 3 X_{B}$ in Section 2.2 which describes an ordinary heterotic string compactification on $T^{2}$ with $S U(16) \subset S O$ (32) gauge symmetry. Note that going to the mirror K3 is necessary to describe the singularity structure of $X_{B}$ in terms of Kähler blow-ups corresponding to holomorphic curves. The polyhedron $\Delta_{B}^{*}$ of $X_{B}^{*}$ is shown on the left-hand side of Fig. $2{ }^{12}$

The shaded triangle represents the hypersurface $\Delta_{E}^{*}$ corresponding to the elliptic fiber of $X_{B}^{*}$. The gauge group $S U(16)$ appears via its affine Dynkin diagram $\hat{A}_{15}$ composed of the points in $\Delta_{B}^{*}$ above the hyperplane $\Delta_{E}^{*}$. There is a second hyperplane $\Delta_{\bar{E}}^{*}$ corresponding to a second elliptic fibration shown in the lower part of Fig. 2. In this case we

\footnotetext{
12 The dark shaded plane divides the polyhedron into two identical halves; only details of the upper half have been displayed.
} 
read off the gauge group $E_{7} \times E_{7}$. This of course reflects the fact that in a toroidal compactification the $E_{8} \times E_{8}$ string lies in the same moduli space as the $S O(32)$ string. ${ }^{13}$ Both elliptic fibrations are of the generic type with $\Gamma_{0}$ monodromy.

As explained in Section 2.2, we can get a K3 $X_{C}$ with $\Gamma_{1}(2)$ monodromy by a blowup of the locus $y=x=0$ in $p_{B}$. This operation corresponds to a $\mathbb{Z}_{2}$ modding of the K3. The action on the mirror polyhedron $\Delta_{B}^{*}$ is shown on the right-hand side of Fig. 2 . It acts as a $\mathbb{Z}_{2}$ on the homology lattice which freezes the volumes of pairs of spheres in the $\hat{A}_{15}$ diagram to the same values. Points in $\Delta_{C}^{*}$ which correspond to a pair of spheres are denoted by a black node in Fig. 2. The modding folds the Dynkin diagram of $S U(16)$ to that of $S p(8)$ and similarly that of $E_{7} \times E_{7}$ in the second elliptic fibration to $F_{4} \times F_{4}$. The $S p(8)$ singularity corresponds to the gauge symmetry of the eight-dimensional CHL vacuum. The $F_{4} \times F_{4}$ singularity appears in six dimensions upon compactification of the eight-dimensional CHL vacuum on a $T^{2}$ with Wilson line [13]. We now turn to a discussion of how the maximal dimension of a vacuum can be determined from the geometric data.

\subsection{Identifying F-theory limits}

To establish the existence of a higher than six-dimensional vacuum, we have to show that it is possible to find an appropriate limit where the fiber of a K3 $X_{2}^{(i)}$ shrinks to zero size. This limit corresponds to a decompactification of the heterotic string on $T^{4}$. From the point of view of modding the type IIA theory compactification by automorphism of $\mathrm{K} 3$ it is clear that the possibility to decompactify some of the circles of the heterotic dual on $T^{4}$ implies the existence of a sublattice $\Gamma_{k, k}$ in the homology of $\mathrm{K} 3$ which is invariant under the automorphism. Moreover, if we use a direction $\Gamma_{k, k}$ on which the automorphism acts as a shift, though we can decompactify in this direction, the automorphism becomes trivial in the large radius limit and we land back on a conventional vacuum without a modding.

As for the first condition, to have an invariant $\Gamma_{k, k}$ lattice, it is fulfilled by construction for the elliptically fibered $\mathrm{K} 3$ manifolds with $\Gamma_{1}(n)$ monodromies that we discuss. The question of whether there is a shift action on the heterotic $T^{2}$ that corresponds to the elliptic fiber can also be read off from the geometric data: if all points in the hyperplane $\Delta_{E}^{*}$ that describes the elliptic fiber represent a single two sphere in $X_{2}^{(i)}$, then there is no shift action on the dual $T^{2}$ and we can decompactify without interfering with the action of the modding. If there are points in $\Delta_{E}^{*}$ which represent $n$ spheres, there is an $n$th order shift symmetry acting on the dual $T^{2}$.

\subsection{1. $\Gamma_{\mathrm{I}}$ (2) monodromy: a 9 d $C H L$ vacuum}

In the first fibration with $S p(8)$ gauge symmetry, the points in the hyperplane $\Delta_{E}^{*}$ correspond to single curves in $X_{C}^{*}$. We can contract the fiber and get the dual of the eight-

\footnotetext{
${ }_{13}^{3}$ In particular these two fibrations are inherited from the two fibrations of the K3 with maximal singularity $E_{8} \times E_{8}$ or $S O(32)\lceil 27\rceil$.
} 
dimensional CHL vacuum. This is in agreement with the action of the $\mathbb{Z}_{2}$ automorphism described in $[25,15]$, which leaves invariant a $\Gamma_{4,4}$ lattice with no shifts in a $\Gamma_{3,3}$ part. Thus, we can actually decompactify to nine dimensions [28].

On the other hand, for the second fibration with $F_{4} \times F_{4}$ gauge symmetry we observe that the point representing the section has multiplicity two corresponding to an order two shift on $T^{2}$. This is in agreement with the fact that in order to get $F_{4} \times F_{4}$ we had to switch on a Wilson line on a further circle starting from an eight-dimensional CHL compactification [13].

\subsection{2. $\Gamma_{1}(3)$ monodromy: a $7 d$ CHL vacuum}

A polyhedron in the moduli space with $\Gamma_{1}(3)$ monodromy is shown in Fig. 3. It describes the dual to a heterotic compactification with rank reduction -12 and $G_{2} \times G_{2}$ gauge symmetry. Note that in this case we have only one elliptic fibration and moreover there is a point of order three in the hyperplane $\Delta_{E}^{*}$ corresponding to a order three shift on the torus of the heterotic theory. Therefore we cannot decompactify without destroying the CHL modding; rather we flow to a point in the moduli space of ordinary heterotic compactification with $S O(8)^{2}$ gauge symmetry in this limit. This is in agreement with the $\mathbb{Z}_{3}$ action described by Nikulin, which leaves invariant a $\Gamma_{2,2}$ lattice but acts as a shift on a $\Gamma_{1,1}$ part of it.

\subsection{3. $\Gamma_{1}(4)$ monodromy}

Since the $\mathbb{Z}_{4}$ action leaves a $\Gamma_{3,3}$ torus invariant but acts as a shift on a $\Gamma_{1,1}$ sublattice, we are lead to conclude, in analogy with the cases considered above, that we can decompactify to eight dimensions. However, due to the fact that the elliptic fiber is represented as a complete intersection, the description of the structure of the sections and the singularities on them is not straightforward. Hence it is hard to distinguish whether this is a new $8 \mathrm{~d}$ theory or whether it merely flows in the F-theory limit to a special point in the moduli space of an already known one.

\subsection{Frozen moduli spaces in toric geometry}

We have seen that the toric geometries reproduce quite well the moduli spaces of reduced rank of the heterotic theory. We will give now a more precise formulation of this fact using some more technology of toric geometry. In fact, there is a well-known phenomenon in Calabi-Yau manifolds constructed as hypersurfaces in toric varieties which has been considered mostly as a technical subtlety so far: the appearance of socalled non-toric divisors and non-polynomial deformations. This situation refers to the fact that a given toric representation of a Calabi-Yau manifold $X$ might not allow for a general deformation but some of the Kähler or complex structure moduli are frozen to a specific value. What we suggest is that rather than being a technical subtlety, these restricted Calabi-Yau moduli spaces are in one-to-one correspondence with physical moduli spaces, where some moduli are stuck due to background fields. As was described in Section 3.1 we obtain a $\mathrm{K} 3$ with $\Gamma_{1}(n)$ monodromy from a $\mathbb{Z}_{n}$ modding. This modding 
removes points from the dual polytope $\Delta^{\star}$ of $X$, reducing the rank of the gauge group. In $\Delta$ this has the effect of introducing so called non-toric deformations. Although the K3 still has a total of 20 Kähler and complex structure deformations, a certain number of them cannot be represented toricly. That is from a toric description the sizes of twocycles are set to identical values. ${ }^{14}$ It is this frozen F-theory geometry which accounts for the reduction of rank leading to the $\mathrm{CHL}$ dual.

In the conventional heterotic case, with vector structure, the total number of $\mathrm{K} 3$ deformations from both, Kähler and complex deformations, adds up to $\operatorname{dim} H_{1,1}(K 3)=$ 20 corresponding to the Narain lattice $\Gamma_{18,2}$. Reducing the rank of the gauge group in the fibration by reducing the number of points of a dual polyhedron $\Delta^{\star}$, or equivalently the number of Kähler deformations, is compensated by the correct number of new complex deformations corresponding to new points in the polyhedron $\Delta$ describing the mirror $\mathrm{K} 3$. However, in the present case, the reduction of Kähler moduli is not compensated by the complex deformations leading to a reduced number of $\mathrm{K} 3$ moduli.

Let us review briefly the appearance of non-toric and non-polynomial deformations.

An $n$-dimensional toric hypersurface $X_{n}(\Delta)$ is defined as the zero locus of the section of an appropriate line bundle on a non-singular toric variety $X(\Sigma)$. The $n+1$-dimensional toric variety $X(\Sigma)$ is defined as the quotient of $\mathbb{C}^{N} \backslash F$ by $\left(\mathbb{C}^{\star}\right)^{N-n-1}$. If $x_{i}$ are coordinates on $\mathbb{C}^{N}$, the disallowed set $F$ is given by subsets $\bigcap_{k} x_{i_{k}}=0$ related to fixed points of the scaling actions. The simplest example is $\mathbf{P}^{N}$ with $N+1$ coordinates $x_{i}$, one $\mathbb{C}^{\star}$ action $x_{i} \rightarrow \lambda x_{i}$ with the fixed point $x_{i}=0 \forall i$ omitted.

The toric divisors $D_{i}: x_{i}=0$ generate a basis for $H^{n}(X(\Sigma))$. Given a representation $X_{n}(\Delta)$ of a Calabi-Yau manifold $X_{n}$ as a hypersurface in $X(\Sigma)$, the toric divisors $D_{i}$ descend to elements $D_{i}^{X}$ in $H^{n-1}\left(X_{n}(\Delta)\right)$ from the intersection $D_{i} \cap X$. However, if it happens that the intersection $D_{i} \cap X_{n}(\Delta)$ contains $l$ disconnected components $D_{i}^{X, \alpha}, \alpha=$ $1, \ldots, l$ in $X_{n}$, a priori independent elements of $H^{n-1}\left(X_{n}\right)$ are represented by the same class $D_{i}$ in the ambient space. As a consequence, the a priori different volumes of the divisors $D_{i}^{X, \alpha}$ are frozen to the same value. Thus, the hypersurface $X_{n}(\Delta)$ can only represent a restricted subset of the Calabi-Yau moduli space $X_{n}$.

To a hypersurface $X_{n}(\Delta)$ defined as a hypersurface in a toric variety corresponds a polyhedron $\Delta$ of lattice points in a standard lattice $M \sim \mathbb{Z}^{n+1}$; c.f. the discussion in Section 2.2. In particular, the polyhedron $\Delta$ encodes in a very efficient way information about the cohomology and the intersection properties of $X_{n}(\Delta)$. Moreover, there is a canonical construction of the mirror manifold $X_{n}^{\star}\left(\Delta^{\star}\right)$ of $X_{n}(\Delta)$ in terms of the dual polyhedron $\Delta^{\star}$. In [29] Batyrev shows that the Hodge numbers $h^{1,1}$ are determined by the polyhedron via

$$
\begin{aligned}
h^{1,1}\left(X_{n}(\Delta)\right) & =h^{n-1,1}\left(X_{n}^{\star}\left(\Delta^{\star}\right)\right) \\
& =l\left(\Delta^{*}\right)-(n+2)-\sum_{\text {codim } S^{\star}=1} l^{\prime}\left(S^{\star}\right)+\sum_{\text {codim } S^{*}=2} l^{\prime}\left(S^{\star}\right) \cdot l^{\prime}(S)
\end{aligned}
$$

\footnotetext{
${ }^{14}$ An example of this phenomenon is that of type IIA compactified on the elliptically fibered Calabi-Yau three-fold with base $F_{2}$ in which there is one non-toric deformation. This accounts for the strongly coupled $S U(2)$ with a massless adjoint.
} 
and similarly for $h^{n-1,1}\left(X_{n}(\Delta)\right)=h^{1,1}\left(X_{n}^{\star}\left(\Delta^{\star}\right)\right)$ with the roles of $\Delta$ and $\Delta^{*}$ exchanged. Here $S$ denotes faces of $\Delta$ and $S^{\star}$ the dual face of $S$. $l$ and $l^{\prime}$ are the numbers of integral points on a face and in the interior of a face, respectively. The last term is a correction term corresponding to the non-toric deformations. We will be mainly interested in the cases $n=2,3$. For K3, $h^{1,1}$ gives the rank of the Picard lattice of $X_{2}(\Delta)$ and a non-zero correction corresponds to having simultaneously points on a one-dimensional edge $S$ of the three-dimensional polyhedron $\Delta$ and on the dual edge $S^{*}$ in $\Delta^{*}$. In the 3-fold case, a non-zero correction term arises from having simultaneously points on a face(edge) $S$ of $\Delta$ and the dual edge (face) $S^{*}$ of $\Delta^{*}$.

In general, if we freeze the volumes of two, say, two-cycles to the same value, nothing interesting happens. However, the existence of a hypersurface $X_{n}^{\prime}\left(\Delta^{\prime}\right)$ with restricted moduli space corresponds to the fact that apart from the volumes, these two-cycles share identical intersection properties. In this case we are at a point with extra symmetry and $X_{n}^{\prime}\left(\Delta^{\prime}\right)$ corresponds to the modding of this symmetry. Note that in general we do not expect the special locus in the moduli space to correspond to a singular manifold, but rather to a particularly symmetric configuration. This is analogous to the fact that in the heterotic theory the CHL construction requires the presence of several gauge group factors broken in an identical manner, but not a restoration of non-abelian gauge symmetries.

\subsection{Heterotic compactifications to six dimensions with $U$ (1) backgrounds}

So far we have interpreted the restricted K3 moduli spaces in terms of type IIA compactifications. However, we could also ask about their meaning if we choose to compactify a heterotic string to get an $N=1$ six-dimensional vacuum. Anomaly free $N=$ 1 theories in six dimensions have been described in [30]. A perturbative vacuum requires a combination of abelian and non-abelian gauge instantons on $\mathrm{K} 3$ of total instanton number 24. In addition, minimization of the action of instantons on $\mathrm{K} 3$ requires [31]

$$
F_{i j}=F_{i j}=g^{i j} F_{i j}=0,
$$

implying

$$
\int_{X_{2}} J \wedge \operatorname{tr} F=0 .
$$

The latter equation implies a non-trivial interplay of abelian instantons and $\mathrm{K} 3$ moduli; in particular, decomposing the $U(1)$ flux in harmonic $(1,1)$ forms $\omega^{\mu}$ on $X_{2}, F^{(a)}=$ $\alpha_{\mu}^{(a)} \omega^{\mu}$, and similarily for the Kähler form, $J=t_{\mu} \omega^{\mu}$, Eq. (3.3) gives a linear constraint on the Kähler moduli

$$
\alpha_{\mu}^{(a)} t_{\nu} C^{\mu \nu}=0
$$

where $C^{\mu \nu}$ is the intersection form on $X_{2}$. This constraint leads to vacua with frozen Kähler moduli. Note that we cannot simply restrict to a frozen configuration in a 
generic K3 moduli space, but we should provide a consistent special geometry based only on the remaining perturbations. Moreover, the freezing should involve full vector multiplets, rather than only their scalar components. By duality, this freezing should correspond again to F-theory compactification on a Calabi-Yau manifold with restricted moduli space in hypermultipets. Therefore we arrive at the picture that the heterotic string on a $\mathrm{K} 3 X_{2}$ with frozen moduli due to $U(1)$ instanton background is dual to F-theory on $X_{3}$ with non-polynomial deformations.

Note that from the F-theory point of view, CHL vacua and six-dimensional $U(1)$ instantons are on a very similar footing due to the fact that both bundle moduli and geometric moduli of the heterotic string get mapped to geometric data of the F-theory compactification.

Type IIA compactification on Calabi-Yau three-folds with special fiber models for the elliptic fiber has been considered in [32-35]. However, these models can generically not be pushed to a six-dimensional F-theory compactification dual to heterotic theory with $U(1)$ backgrounds due to the generic monodromy of the elliptic fibration. ${ }^{15}$ Most of these models correspond rather to five-dimensional compactifications with $U(1)$ Wilson lines on an extra circle and therefore flow to the moduli space of heterotic compactifications without $U(1)$ backgrounds in six dimensions. In fact this is clear from the presence of the corresponding transitions between Calabi-Yau manifolds [33]. Note that analysis of the four-dimensional supergravity spectrum cannot tell the difference between a five-dimensional compactification with $U(1)$ Wilson lines and a six-dimensional compactification with $U(1)$ instantons. The difference is precisely detected by the moduli frozen by the constraint (3.3) and severely restricts the possible vacua of this type.

\subsection{Enriques involution and $C H L$ orbifold}

The F-theory dual of a rank $r$ CHL compactification involves naturally a restricted family of elliptic $K 3$ surfaces $X$, whose Picard lattice has rank $\rho=20-r$. The complex structure moduli space will then be of the general form of a locally symmetric space [36]

$$
\mathcal{M}=\Gamma_{T} \backslash O(2,20-\rho) / O(2) \otimes O(20-\rho),
$$

identified by discrete automophisms $\Gamma_{T}$ of the transcendental lattice $T$, which has signature $(2,20-\rho)$. In particular, for the $\mathbb{Z}_{2}$ CHL construction in $8 d$, in the following just denoted by CHL, we have argued that the required $\Gamma_{1}(2)$ monodromy restricts the dimension of the complex structure moduli space to ten and found, besides the hyperbolic factor $H$ required by the fibration structure, further eight algebraic curves in the $A_{1}^{8}$.

There is a well-known example of $K 3$ surfaces whose Picard group has rank ten, namely the double cover of an Enriques surface. Every Enriques surface $Y$ has a $K 3 X$ as an unbranched double cover and can thus be given as $Y=X / \sigma$ with $\sigma$ fixed-point free. The $(2,0)$ form is anti invariant under $\sigma$ and therefore $c_{1}(Y) \neq 0$ is a torsion

\footnotetext{
${ }^{15}$ Our conclusion concerning this point is different from the one suggested in [34].
} 
class: $c_{1}^{2}(Y)=0 . Y$ has no holomorhic periods, but $X$ can be used to define its period map and local and global Torelli theorems have been proven from this for $Y$ [37], see also Ref. [36]. One can identify the induced action of the covering involution $\sigma$ on the cohomology lattice

$$
A(X)=\left(-E_{8}\right) \oplus\left(-E_{8}\right) \oplus H \oplus H \oplus H,
$$

as $\rho\left(x \oplus y \oplus z_{1} \oplus z_{2} \oplus z_{3}\right)=\left(y \oplus x \oplus-z_{1} \oplus z_{3} \oplus z_{2}\right)$ after choosing a compatible marking $\phi: H^{2}(X, \mathbb{Z}) \rightarrow A$, i.e. with $\phi \circ \sigma^{*}=\rho \circ \phi . H$ is the hyperbolic lattice spanned by $e_{1}$, $e_{2}$ with $e_{1} \cdot e_{2}=1 e_{1}=e_{2}^{2}=0$ and $E_{8}$ denotes the $E_{8}$ lattice. Under $\rho, A$ splits

$$
\Lambda=A^{+} \oplus \Lambda^{-}
$$

into an invariant part $\Lambda^{+}=\left(-2 E_{8}\right) \oplus 2 H$, which comes from the Picard lattice $S$ of $X$ and the non-invariant part $A^{-}=\left(-2 E_{8}\right) \oplus 2 H \oplus H$, which comes from the transcendental lattice $T$ in the cohomology of $X$. The cohomology lattice of $Y$ is isometric to $\Lambda^{0}=\Lambda^{+} / 2=\left(-E_{8}\right) \oplus H$. Since $\omega \in A^{-}$and the period integral $([\omega], \gamma)=\int_{\gamma} \omega$ is invariant under $\sigma$ it must vanish for $\gamma$ Poincare dual to an element of $\phi^{-1}\left(A^{+}\right)$. The period points, describing the complex moduli space of $X$ all belong to $\Omega^{-}=\{[\omega] \epsilon$ $\left.\mathbf{P}\left(\Lambda^{-} \otimes \mathbb{C}\right) \mid(\omega, \omega)=0,(\omega, \bar{\omega})>0\right\}$, which contains two disjoint copies of a $10 \mathrm{~d}$ bounded domain as in (3.5), ${ }^{16}$ where the discrete group $\Gamma_{A^{-}}$is the restriction to $A^{-}$of those elements $g$ in $A u t(A)$ which are compatible with (3.7), i.e. $g \circ \rho=\rho \circ g$. This is very close to the description of the moduli space for the CHL in [13], namely (3.5) with $A^{-}$replaced by the invariant part of the Narain lattice $A^{\mathrm{CHL}}=\left(-2 E_{8}\right) \oplus H \oplus H$ under the CHL involution. In fact the difference is the scale 2 on one of the hyperbolic factors due to the $\left(z_{2}, z_{3}\right)$ exchange in $\rho$, which has no analog in the CHL modding. However, Nikulin describes in $[38,39]$ a closely related $\mathbb{Z}_{2}$ operation $\sigma_{N}$ under which the $(2,0)$ form is likewise anti-invariant but, as $\left(z_{2}, z_{3}\right)$ is not exchanged, has now indeed $T=\Lambda_{N}^{-}=\left(-2 E_{8}\right) \oplus H \oplus H$, while $S=\Lambda_{N}^{+}=\left(-2 E_{8}\right) \oplus H . \sigma_{N}$ is not fixed-point free, but fixes two elliptic fibres.

Horikawa [37] gives a construction of $X$ as double covering over $F_{0}=\mathbf{P}^{1} \times \mathbf{P}^{1}$ branched at $B$, a bi-degree $(4,4)$ hypersurface. The generic $B$ has 25 perturbations, seven reparametrisations have to be subtracted, which yields 18 complex structure deformations for $X$. The $\mathbb{Z}_{2}$ involution $\hat{\sigma}$ acts on the coordinates of $F_{0}$ as $\hat{\sigma}:(x: z, u$ : $v) \mapsto(x:-z, u:-v)$. The involution $\sigma$ is the combination of $\hat{\sigma}$ with the covering involution $y \mapsto-y$. We have $13 \sigma$ invariant monomials: $x^{4-2 i} z^{2 i} u^{4-2 j} v^{2 j}, i, j=0,1,2$ and $x^{3-i} z^{1+i} u^{3-j} v^{1+j}, i, j=0,2$. The $S L(2, \mathbb{C})^{2}$ of the two $\mathbf{P}^{1}$ 's is broken to two parameters and the overall scaling removes another parameter. Hence we have ten complex structure deformations. This $X$ has a nice toric description by the polyhedra $\left(\Delta, \Delta^{*}\right)$, which are shown in Fig. 4. $X$ admits at least two and $X^{*}$ at least eight different elliptic fibrations, as inspection of the toric diagram shows. We pick the one in which the torus is represented by $y$ as the double cover of a quartic in $x, z$ and in which $u, v$ become

\footnotetext{
${ }^{16}$ In fact for $Y$ not all of (3.5) is mapped out by period points. It is known that precisely the hyperplanes $H_{d}=\left\{|\omega| \in \Omega^{-} \mid(w, d)=0\right\}$ with $(d, d)=-2$ have to be subtracted $[37,36 \mid$.
} 


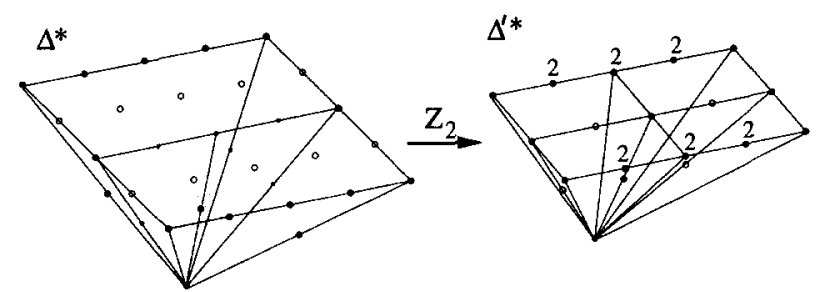

$\Delta$

$\Delta^{\prime}$
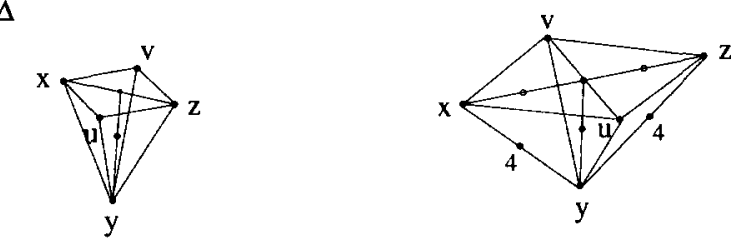

Fig. 4. $\mathbb{Z}_{2}$ modding of a rank(Pic) $=18$ elliptic fibration $\mathrm{K} 3$ to the $\operatorname{rank}(\mathrm{Pic})=10 \mathrm{CHL}$ dual.

the base coordinates. Now we mod out the $\mathbb{Z}_{2}^{\mathrm{CHL}}$, which was described in [26], namely the elliptic involution $y \mapsto-y$, combined with inversion of the inhomogeneous $\mathbf{P}^{\mathbf{l}}$ coordinate $w \mapsto \frac{1}{w}$, which acts in diagonalized form as $v \mapsto-v$. On the other hand $\sigma_{N}$ is identified with $\sigma_{N}:(x: z, u: v) \mapsto(x: z, u:-v)$ with the two fixed fibers located over $(u, v)=(1,0),(0,1)$. The elliptic involution that we do in addition in $\mathbb{Z}_{2}^{\mathrm{CHL}}$ to keep the holomorphic form, exchanges $\Lambda_{N}^{+}$with $\Lambda_{N}^{-}$, hence what we keep is $\Lambda_{N}^{-}=\Lambda^{\mathrm{CHL}}$. The relation between the $\mathrm{K} 3 X_{N}$ admitting $\sigma_{N}$ as involution to the double cover of Enriques surfaces has been pointed out in [39] (see Section 6, example 3). The mirror operation identifies $T\left(X^{*}\right)=2 H \oplus S(X)$ and $S\left(X^{*}\right)=T\left(X^{*}\right)^{\perp}=2 H \oplus\left(-2 E_{8}\right)$ hence it is $X_{N}^{*}$ that is related to the double covering of an Enriques surface.

The orbifold $X / \mathbb{Z}_{2}^{\mathrm{CHL}}$, a $\operatorname{rank}(\mathrm{Pic})=10 \mathrm{~K} 3$, can be readily defined by the reflexive pair $\left(\Delta^{\prime}, \Delta^{* \prime}\right)$, which is obtained, as in [26], from $\Delta^{*}$ by keeping only solid points, which correspond to the invariant monomials, see Fig. 4 . This orbifold has two $A_{1}^{4}$ singularities on two different sections, as can be seen from the points of multiplicity 4 in $\Delta^{\prime}$. Also note that $X^{*}$ has an $S p(4)^{2}$ gauge symmetry.

Finally note that by a series of blow-ups and blow-downs, all within the class of $\operatorname{rank}(10) \Gamma_{1}(2) \mathrm{K} 3$ 's, we can recover the $S p(8)$ case discussed in Section 3.2. The corresponding blow ups take place by adding and removing points in the $(x z u v)$-plane of $\Delta^{\prime}$. Below we indicate the gauge groups which occur in this process. Fixed are the points $x, z$ and the configuration ( $x z u v$ ) is the starting configuration with $S p(4)^{2}$ from Fig. 4 . One gets $(u \hat{u} v): S p(3) \times S p(4),(u \hat{u} v \hat{v}): S p(2) \times S p(4),(\hat{u} v \hat{v}): S p(2) \times S p(5),(\hat{u} \hat{v})$ : $S p(2) \times S p(6),(\hat{u} \hat{v} \hat{v}) S p(6),(\hat{u} \hat{\hat{u}} \hat{\hat{v}}) S p(7)$ and $(\hat{\hat{u}} \hat{\hat{v}})$ gives the $S p(8)$ we discussed in Section 3.2. 


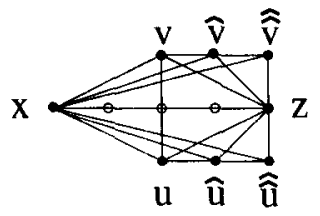

Fig. 5. Elementary transformations within the $\Gamma_{1}(2) \mathrm{K} 3$.

\section{Moduli space of $\boldsymbol{G}$ bundles without vector structure on elliptic curves}

Having identified F-theory geometries corresponding to heterotic CHL models in eight dimensions, leads a very nice application for the description of the moduli space of $G$ bundles over elliptic curves. In [11] it was shown that CHL string constructions in eight dimensions can be interpreted as heterotic compactifications on $T^{2}$ with a vector bundle without vector structure. For example, for the heterotic theory with gauge group $G=\operatorname{Spin}(32) / \mathbb{Z}_{2}$ in ten dimensions, it is consistent to have backgrounds obstructing the presence of the vector representation of $S O(32)$, due to the absence of perturbative states in this representation. The non-triviality of the action on the vector representation is measured by a mod two cohomology class $\tilde{w}_{2}$ which integrates to -1 on a two-cycle on which vector structure is obstructed.

We are interested in the moduli space of such bundles over the elliptic curve $E$ on which the heterotic string is compactified. These moduli spaces turn out to be described by weighted projective spaces [40] and have been analyzed in detail in the mathematical literature [41]. In [23] it was realized that one can get a very simple and canonical description of these moduli spaces in terms of complex geometries as a consequence of heterotic/type IIA duality together with mirror symmetry. A simple reasoning goes as follows: F-theory on elliptically fibered $\mathrm{K} 3$ with a singularity $H$ from collisions of singular fibers is dual to heterotic string on $E$ with a vector bundle taking values in $G$, the commutant of $H$ in the primordial gauge group $E_{8} \times E_{8}$ or $S O(32)$. On compactification on a further $T^{2}$ to six dimensions, we blow up the $H$ singularity in Kähler moduli and obtain type IIA on the smooth $\mathrm{K} 3 X(H)$, dual to heterotic theory on $T^{2} \times T^{2}$, where now we have in addition to the $G$ Wilson lines on the first $T^{2}$ also $H$ Wilson lines on the second torus, breaking to the generic abelian gauge group $U(1)^{24}$. Thus, the moduli space of Kähler blow-ups of the $H$ singularity in the $\mathrm{K} 3 X(H)$ is isomorphic to the moduli space of $H$ Wilson lines on the second $T^{2}$. Application of mirror symmetry gives type IIA on the mirror $\mathrm{K} 3 X(G)$, with the roles of complex and Kähler moduli exchanged. Thus, the complex deformations of $X(G)$ give a realization of the moduli space of $H$ bundles on $T^{2}$.

Let us apply this procedure to the $\mathrm{K} 3$ dual to heterotic CHL string in eight dimensions. The only difference compared to [23] is that we have to apply mirror symmetry to the local $S p(8)$ singularity with symmetric fiber. After a change of variables, the defining equation for $X$ can be rewritten as

$$
p=a_{0} v^{-1}+\left(y^{2}+x^{4}+z^{4}+\alpha y x z\right)+v\left(z^{8}+z^{7} x+\ldots+x^{8}\right)=0 .
$$


To concentrate on a neighborhood of the local singularity mirror to the $S p(8)$ singularity in the elliptic fiber of the mirror $\mathrm{K} 3$, we have to set the coefficient $a_{0}$ of the $v^{-1}$ term to zero. In this limit, we can integrate out $v$ and obtain the spectral cover description of [41]:

$$
p_{E}=y^{2}+x^{4}+z^{4}+\alpha y x z=0, \quad p_{C}=z^{8}+z^{7} x+\ldots+x^{8}=0 .
$$

These equations describe eight points given by the zeros of $p_{C}$ on the elliptic curve defined by $p_{E}=0$. Actually, since there is no $y$ dependence in $p_{C}$, we have to divide by the $\mathbb{Z}_{2}$ operation $y \rightarrow-y$ and get eight points on the orbifold $T^{2} / \mathbb{Z}_{2}$. This is the usual description of $S p(8)$ bundles on an elliptic curve $E$ [41]. However, since the torus $p_{E}$ is not of the generic form, we have in addition to take into account the shift symmetries $(2.5)$; this gives further identifications on $T^{2} / \mathbb{Z}_{2}$ by the two half-period shifts $z \sim z+\frac{1}{2}, z \sim z+\frac{\tau}{2}$ in a lattice basis with periods $(1, \tau)$. Therefore the points $p_{C}=0$ are defined on a orbifold $T^{2} / \mathbb{Z}_{2}$ with the torus lattice rescaled by a factor two in each direction. This agrees perfectly with the description in Ref. [11], where it has been argued via different reasoning that the dual orientifold lives on a half-sized torus. This gives a further independent check on our identification of the F-theory geometry.

The above description can be easily generalized to arbitrary $S p(n)$ bundles using the local mirror constructions of [23] with a symmetric torus $E^{(2)}$ as the generic elliptic fiber of the elliptic singularity. It would be interesting to work out a more complete description for other gauge groups as well as for the other tori $E^{(3)}$ and $E^{(4)}$.

\section{Acknowledgements}

We would like to thank D. Husemöller, W. Lerche, D. Lowe, D. Morrison, J. Polchinski, H. Skarke, S. Stieberger and A. Todorov for valuable discussions. The work of P.B. and P.M. was supported in part by the Natural Science Foundation under Grant No. PHY94-07194. S.T. is supported by GIF, the German-Israeli Foundation for Scientific Research and by the European Commission TMR programme ERBFMRX-CT96-0045. A.K., P.M. and S.T. thank the ITP in Santa Barbara for hospitality.

\section{Appendix A. Congruence subgroups $\Gamma_{1}(n)$ and $\Gamma(n)$}

Let us collect some relevant properties of the subgroups $\Gamma(N), \Gamma_{1}(N), \Gamma_{0}^{0}(N)$, $\Gamma_{0}(N)$ of $\Gamma_{0}=S L(2, \mathbb{Z})$. More details can be found in [42-44]. The definitions of the above subgroups are

$$
\begin{aligned}
& \Gamma_{0}(n)=\left\{\left(\begin{array}{ll}
a & b \\
c & d
\end{array}\right) \in \Gamma_{0}, c \equiv 0 \bmod n\right\}, \\
& \Gamma_{0}^{0}(n)=\left\{\left(\begin{array}{ll}
a & b \\
c & d
\end{array}\right) \in \Gamma_{0}, b \equiv c \equiv 0 \bmod n\right\},
\end{aligned}
$$




$$
\begin{aligned}
\Gamma_{\mathrm{l}}(n) & =\left\{\left(\begin{array}{ll}
a & b \\
c & d
\end{array}\right) \in \Gamma_{0}, a \equiv d \equiv 1 \bmod n, c \equiv 0 \bmod n\right\}, \\
\Gamma(n) & =\left\{\left(\begin{array}{ll}
a & b \\
c & d
\end{array}\right) \in \Gamma_{0}, a \equiv d \equiv 1 \bmod n, b \equiv c \equiv 0 \bmod n\right\} .
\end{aligned}
$$

The corresponding inhomogeneous groups are denoted by $\bar{\Gamma}_{0}$, etc. From its definition we see that $\Gamma_{0}(n)$ fixes the $n$ order $n$ points $(k / n, 0) \bmod (r, s), k=0, \ldots, n-1, r, s \in \mathbb{Z}$ (as a set), while $\Gamma_{0}^{0}(n)$ fixes the $n$ points $(k / n, 0) \bmod (r, s), k=0, \ldots, n-1, r, s \in \mathbb{Z}$ and the $n$ points $(l / n, 0) \bmod (r, s), k=0, \ldots, n-1, r, s \in \mathbb{Z} . \Gamma_{1}(n)$ fixes the $n$ points $(k / n, 0) \bmod (r, s), k=0, \ldots, n-1, r, s \in \mathbb{Z}$ pointwise, whereas $\Gamma(n)$ fixes the $n^{2}$ $n$-division points $(k / n, l / n) \bmod (r, s), k, l=0, \ldots, n-1, r, s \in \mathbb{Z}$ pointwise. For the elliptic curve these coordinates refer to the basis spanned by the periods $\left(\omega_{1}, \omega_{2}\right)$.

A special case appears for $n=0 \bmod 2$, since in this case the points of order two are preserved, which are the branch points of the double covering of the Riemann sphere. By $\wp\left(\frac{\omega_{i}}{2}\right)=e_{i}$ with $\omega_{i}=\omega_{1}, \omega_{2}, \omega_{1}+\omega_{2}$ these points are identified with the roots of the Weierstrass form $\left(\wp^{\prime}\right)^{2}=4 \prod_{i=1}^{3}\left(\wp-e_{i}\right)$ for $E$ and each invariant root gives rise to a global section of the elliptic fibration. For $\Gamma_{0}(2 n)$ we have (at least) two global sections, the infinity section $y=\wp^{\prime}=\infty, x=\wp=\infty$ corresponding to $(0,0)$ as well as a further global section $\left(\frac{1}{2}, 0\right)$, whereas for $\Gamma_{0}^{0}(2 n)$ we have four global sections corresponding to the four branch points $(0,0),\left(\frac{1}{2}, 0\right),\left(0, \frac{1}{2}\right),\left(\frac{1}{2}, \frac{1}{2}\right)$.

Normal forms and restricted monodromies

In this appendix we summarize the torsion structure, the degenerations and the monodromies which occur for the fibrations $X_{2}^{(2)}, X_{2}^{(3)}$ and $X_{2}^{(4)}$ defined in Section 2.

First we relate the forms of our equations to the forms used in Table 3 of [45] for the parametrization of torsion structures of order $2,3,4$

$$
\begin{gathered}
X_{2}^{(2)}: y^{2}=x\left(x^{2}+a x+b\right), \\
a=2 f_{4}, \quad b=f_{4}^{2}-4 f_{8} \\
X_{2}^{(3)}: y^{2}+a x y+b y=x^{3}, \\
a=3 f_{2}, \quad b=f_{2}^{3}-27 f_{6} \\
X_{2}^{(4)}: y^{2}+a x y+a b y=x^{3}+b x^{3}, \\
a=4 f_{2}, \quad b=f_{2}^{3}-16 f_{4} .
\end{gathered}
$$

The above rational relation between the parametrisations is most easily verified by bringing both representations of the elliptic curves to the Weierstrass form. It shows that the specialized fibrations have besides the zero section $1,2,3$ sections at $Z_{2}, Z_{3}, Z_{4}$ points.

For the analysis of the degenerations the key data are the orders of the zeros of $g_{2}, g_{3}$ and $\delta=g_{2}^{3}-27 g_{3}^{2}$ in the associated Weierstrass form $y^{2}=4 x^{3}-g_{2} x-g_{3}$ of the elliptic fibration.

$$
X_{2}^{(2)}: g_{2}=f_{4}^{2}+12 f_{8}, \quad g_{3}=\frac{1}{\sqrt{27}}\left(36 f_{8}-f_{4}^{2}\right) f_{4},
$$




\begin{tabular}{|c|c|c|c|c|c|c|c|c|}
\hline$o\left(g_{2}\right)$ & $o\left(g_{3}\right)$ & $o(\delta)$ & fiber & singularity & mon & $X_{2}^{(2)}$ & $X_{2}^{(3)}$ & $X_{2}^{(4)}$ \\
\hline$\geqslant 0$ & $\geqslant 0$ & 0 & smooth & none & $\left(\begin{array}{ll}1 & 0 \\
0 & 1\end{array}\right)$ & + & + & + \\
\hline 0 & 0 & $n$ & $\mathbf{I}_{n}$ & $A_{n-1}$ & $\left(\begin{array}{ll}1 & n \\
0 & 1\end{array}\right)$ & $\begin{array}{c}1-8 \\
10 \ldots . .24\end{array}$ & $\begin{array}{c}1-6 . \\
9, \ldots, 24\end{array}$ & $\begin{array}{l}1-4,6,8 \\
12, \ldots .24\end{array}$ \\
\hline$\geqslant 1$ & 1 & 2 & II & none & $\left(\begin{array}{cc}1 & 1 \\
-1 & 0\end{array}\right)$ & - & - & - \\
\hline$\geqslant 1$ & $\geqslant 2$ & 3 & III & $A_{1}$ & $\left(\begin{array}{cc}0 & -1 \\
1 & 0\end{array}\right)$ & + & - & - \\
\hline$\geqslant 2$ & 2 & 4 & IV & $A_{2}$ & $\left(\begin{array}{cc}0 & 1 \\
-1 & -1\end{array}\right)$ & - & + & - \\
\hline 2 & $\geqslant 3$ & $n+6$ & $\mathrm{I}_{n}^{*}$ & $D_{n+4}$ & $-\left(\begin{array}{ll}1 & n \\
0 & 1\end{array}\right)$ & $0-4$ & - & 1 \\
\hline$\geqslant 2$ & 3 & $n+6$ & $\mathrm{I}_{n}^{*}$ & $D_{n+4}$ & $-\left(\begin{array}{ll}1 & n \\
0 & 1\end{array}\right)$ & - & - & - \\
\hline$\geqslant 3$ & 4 & 8 & $\mathrm{IV}^{*}$ & $E_{6}$ & $\left(\begin{array}{cc}-1 & -1 \\
1 & 0\end{array}\right)$ & - & + & - \\
\hline 3 & $\geqslant 5$ & 9 & III* $^{*}$ & $E_{7}$ & $\left(\begin{array}{cc}0 & -1 \\
1 & 0\end{array}\right)$ & + & - & - \\
\hline$\geqslant 4$ & 5 & 10 & II* & $E_{8}$ & $\left(\begin{array}{cc}0 & -1 \\
1 & 1\end{array}\right)$ & - & - & - \\
\hline
\end{tabular}

$$
\begin{aligned}
\delta & =108 f_{8}\left(4 f_{8}-f_{4}^{2}\right)^{2} \\
X_{2}^{(3)}: g_{2} & =f_{2}\left(f_{2}^{3}+216 f_{6}\right), \quad g_{3}=\frac{1}{\sqrt{27}}\left(f_{2}^{6}-540 f_{6} f_{2}^{3}-5832 f_{6}^{2}\right), \\
\delta & =1728 f_{6}\left(f_{2}^{3}-27 f_{6}\right)^{3} \\
X_{2}^{(4)}: g_{2} & =f_{2}^{4}+224 f_{4} f_{2}^{2}+256 f_{4}^{2}, \\
g_{3} & =\frac{1}{\sqrt{27}}\left(4096 f_{4}^{3}-8448 f_{2}^{2} f_{4}^{2}-528 f_{2}^{4} f_{4}+f_{2}^{6}\right), \\
\delta & =1728 f_{2}^{2} f_{4}\left(f_{2}^{2}-16 f_{4}\right)^{4} .
\end{aligned}
$$

Using Kodaira's results [46] as summarized in the first six columns of the following table, we study the possible degenerations. The results are summarized in the last three columns. For example, for $\mathbf{P}_{1,1,2}^{2}[4]$ for a fiber of type II to be present we need $\delta \sim t^{2}$ which is only possible if (i) $f_{8} \sim t^{2}$ or (ii) $4 f_{8}-f_{4}^{2} \sim t$. For case (i) the condition $g_{3} \sim t$ cannot be satisfied. Case (ii) requires $f_{8} \sim a+b t$ and $f_{4} \sim 4 a+c t$ which contradicts the conditions on $g_{2}$ and $g_{3}$. Thus, the fiber type II must be absent.

Beside showing the possible singularities the analysis allows us to confirm the monodromy groups. In general we know that the monodromy group $\Gamma$ for these cases must satisfy $\Gamma(n) \subseteq \Gamma \subseteq \Gamma_{0}$ with $n=2,3,4$, respectively.

$\Gamma_{0}(2)$ has parabolic vertices and vertices of order 2 but none of order three (see e.g. Ref. [47]). The table shows that the II, $\mathrm{II}^{*}, \mathrm{IV} \mathrm{IV}^{*}$ fibers, which act on $\tau$ with order three, are indeed excluded. Of course the monodromies in Table 1 result from a local analysis and are therefore determined only up to conjugation. However, from the fact that the generated monodromy $\Gamma$ has the property $\Gamma \subset S L(2, \mathbb{Z})$ and contains the shift 
$T$ as well as elements of order 2 but no element of order 3 , we conclude that $\Gamma=\Gamma_{0}(2)$, up to conjugation. If $f_{8}$ factorizes $f_{8}=\hat{f}_{4}^{2}$, the Weierstrass form factorizes completely and odd $T$ shifts as well as order 2 and 3 elements are absent, which shows that the monodromy is $\Gamma(2)$.

$\Gamma_{0}(3)$ has parabolic vertices and vertices of order three and as the generated monodromy $\Gamma$ contains $T$ and elements of order 3, but no element of order 2 , as can be seen from the table, $\Gamma$ must be contained in $\Gamma_{0}(3)$. However, not all singular fibers which are compatible with $\Gamma_{0}(3)$ do actually occur, which signals that the monodromy is $\Gamma_{1}(3)$. If $f_{6}$ factorizes as $f_{6}=\hat{f}_{2}^{3}$, we see only $T^{3 n}$ shifts and neither order 3 nor order 2 elements, hence we get back $\Gamma(3)$.

Finally $\Gamma_{0}(4)$ has only parabolic vertices and again from the table we see that all finite order monodromies are indeed absent, while the $T$ shift is present, leading to the conclusion that the monodromy is contained in $\Gamma_{0}(4)$. If $f_{4}=\hat{f}_{2}^{2}$, the Weierstrass form again completely factorizes. We get no finite order generators, no shift $T$, but $T^{2}$ and hence a monodromy $\Gamma(4) \subset \Gamma \subset \Gamma_{0}(4) . \Gamma(4)$ is only recovered if $f_{4}=f_{1}^{4}$ and $f_{2}=\hat{f}_{1}^{2}$, in which all complex moduli of the compactification are fixed.

\section{References}

[1] C. Vafa, Nucl. Phys. B 469 (1996) 403.

[2] D.R. Morrison and C. Vafa, Nucl. Phys. B 473 (1996) 74.

[3] D.R. Morrison and C. Vafa, Nucl. Phys. B 476 (1996) 437.

[4] P. Candelas and A. Font, Nucl. Phys. B 511 (1998) 296.

[5] M. Bershadsky et al., Nucl. Phys. B 481 (1996) 215.

[6] P. Aspinwall and D.R. Morrison, Nucl. Phys. B 503 (1997) 533.

17] W. Lerche and S. Stieberger, Prepotential, mirror map and F-theory on K3, hep-th/9804176.

181 S. Chaudhuri, G. Hockney and J.D. Lykken, Phys. Rev. Lett. 75 (1995) 2264.

[9] C.M. Hull and P.K. Townsend, Nucl. Phys. B 438 (1995) 109.

|10| A. Sen and S. Sethi, Nucl. Phys. B 499 (1997) 45.

111 E. Witten, Toroidal compactification without vector structure, hep-th/9712028.

1121 M. Bershadsky, T. Pantev and V. Sadov, hep-th/9805056.

[13] S. Chaudhuri and J. Polchinski, Phys. Rev. (1995) 7168.

|14| W. Lerche, R. Minasian, C. Schweigert and S. Theisen, Phys. Lett. B 424 (1998).

115] S. Chaudhuri and D. Lowe, Nucl. Phys. B 459 (1996) 113.

[16] S. Chaudhuri and D. Lowe, Nucl. Phys. B 469 (1996) 21.

[17] A. Sen, Nucl. Phys. B 475 (1996) 562.

[18] Victor V. Batyrev, Duke Math. J. 69 (1993) 349.

119| D. Cox, The homogeneous coordinate ring of a toric variety, alg-geom $/ 9210008$.

[20] I.V. Dolgachev, Mirror symmetry for lattice polarized K3 surfaces, alg-geom/9502005.

$121]$ A. Klemm, W. Lerche and P. Mayr, Phys. Lett. B 357 (1995) 313.

[22] A.C. Avram, M. Kreuzer, M. Mandelberg and H. Skarke, Nucl. Phys. B 494 (1997) 567.

[23] S. Katz, P. Mayr and C. Vafa, Adv. Theor. Math. Phys. 1 (1998) 53.

$124 \mid$ J.H. Schwarz and A. Sen, Phys. Lett. B 357 (1995) 323.

[25] V.V. Nikulin, Trans. Moscow Math. Soc. 2 (1980) 71.

[26] S. Kachru, A. Klemm and Y. Oz, Calabi-Yau Duals for CHL Strings, hep-th/9712035.

[27] P. Candelas and H. Skarke, Phys. Lett. B 413 (1997) 63

|28| J. Park, Phys. Lett. B 418 (1998) 91.

|29| V. Batyrev, J. Alg. Geom. 3 (1994); Duke Math. J. 69 (1993) 349

|30| M.B. Green and J.H. Schwarz, Nucl. Phys. B 254 (1985) 327. 
[31] E. Witten, Nucl. Phys. B 268 (1986) 79.

[32] S. Kachru and C. Vafa, Nucl. Phys. B 450 (1995) 69.

[33] A. Klemm, P. Mayr and C. Vafa, BPS states of exceptional non-critical strings, to appear in the proceedings of the conference Advanced Quantum Field Theory, (in memory of Claude Itzykson), CERN-TH-96-184, hep-th/9607139.

[34] G. Aldazabal, A. Font, L. Ibanez and A.M. Uranga, Nucl. Phys. B 492 (1997) 119

[35] P. Candelas, E. Perevalov and G. Rajesh, Nucl. Phys. B 502 (1997) 594.

[36] W. Barth, C. Peters and A. Van de Ven, Compact Complex Surfaces (Springer, Berlin, 1984).

[37] E. Horikawa, On the periods of Enriques surfaces I, Math. Ann. 234 (1978) 73.

[38] V.V. Nikulin, On the quotient groups of the automorphism groups of hyperbolic forms by the subgroups generated by 2-reflections, J. Sov. Math. 22 (1983) 1401

[39] V.A. Gritsenko and V.V. Nikulin, K3 surfaces, lorentzian Kac-Moody algebras and mirror symmetry, alg-geom/951008.

[40] E. Looijenga, Invent. Math. 38 (1977) 17; 61 (1980) 1.

[41] R. Friedman, J. Morgan and E. Witten, Commun. Math. Phys. 187 (1997) 679.

[42] N. Koblitz, Introduction to Elliptic Curves and Modular Forms (Springer, Berlin, 1984).

[43] D. Husemöller, Elliptic Curves (Springer, Berlin, 1986).

[44] B. Schoeneberg, Elliptic Modular Functions (Springer, Berlin, 1974).

[45] D.S. Kubert, Proc. London Math. Soc. 33 (1976) 193.

[46] K. Kodaira, On compact analytic surfaces, Ann. of Math. 77 (1963) 563.

[47] J.J. Lehner, Discontinuous groups and automorphic functions, Math. Surveys 8, AMS (1982) c1964. 